\title{
Minimizing the number of wavelengths in a cluster WDM mesh- based ONoC through application-specific mapping
}

\author{
Wentao $\mathrm{Xu}{ }^{1,2 a)}$, Ning $\mathrm{Wu}^{1}$, and Fen $\mathrm{Ge}^{1}$
}

Abstract Wavelength division multiplexing (WDM) mesh-based optical network on chip (ONoC) have recently received considerable attention due to their regular topology and higher throughput. However, available wavelengths are finite resources. Thus, to minimize the number of wavelengths, this letter proposes an efficient WDM mesh-based ONoC mapping approach, which is based on a particle swarm optimization algorithm. Experimental results reveal that our proposal has a positive effect in minimizing the number of wavelengths needed compared to random mapping. The tradeoff between the number of wavelengths and the network size is also investigated, and it is found that increasing the network size can reduce the number of wavelengths needed. Meanwhile, the experiments show that the structure has better network performance and less communication resource utilization.

Keywords: optical network on chip, wavelength division multiplexing, ONoC mapping

Classification: Integrated optoelectronics

\section{Introduction}

The on-chip optical communication using waveguides as the transmission medium has essential advantages compared to traditional electrical communication $[1,2,3,4]$. The characteristics of low delay, high bandwidth, and low power consumption can effectively solve the ongoing bottlenecks of on-chip communication $[5,6,7]$.

In the design of on-chip optical networks, the communication architecture is often structured based on optical circuit switching, which causes a blocking problem when the communication links are occupied by different data flows, leading to lower throughput and higher energy consumption $[8,9]$. The wavelength division multiplexing (WDM) technology enables optical signals of multiple wavelengths to be transmitted in the same waveguide, which avoids blocking and effectively improves the communication performance $[10,11,12]$. However, the wavelength is a finite resource. The manufacturing process of silicon-based photonics limits the number of wavelengths available for on-chip communications. Large arrays of fixed-wavelength light sources should be required for more

${ }^{1}$ College of Electronic and Information Engineering, Nanjing University of Aeronautics and Astronautics, Nanjing 210016, China

${ }^{2}$ College of Science, Nanjing University of Aeronautics and Astronautics, Nanjing 210016, China

a)xuwentao@nuaa.edu.cn

wavelengths, and more optical microring resonators (MRs) with different resonance frequencies should be integrated into ONoC [13], which increases the manufacturing cost. When multiple optical signals are transmitted in the same waveguide, crosstalk between the signals inevitably affects the reliability of communication $[14,15,16]$. Thus, it is sensible to minimize the number of used wavelengths when designing a WDM-based on-chip optical network.

The number of wavelengths in the ONoC can be reduced by scheduling method, which allocates wavelengths to communication node pairs in network [17]. However, scheduling method does not consider the assignment of IP cores to network and it is suitable for ring and bus topology, but not useful for mesh topology. Scheduling and mapping are two different stages in the design of ONoC. Mapping determines the core's location in the network [18], it has a significant influence on network performance. For mesh $\mathrm{ONoC}$, there is a close relationship between the number of wavelengths and the mapping of cores for mesh-based ONoC. It is necessary to optimize the utilization of wavelength resources in the mapping process. Many researchers have done lots of work on ONoC mapping. In [19], a mapping method is proposed for the first time in the literature to address the power-loss problem. In [20], Edoardo et al. developed an application mapping tool called PhoNoCMap to find mapping solution. In [21], Lei Guo et al. proposed a mapping methodology for 3D ONoC topology to ensure high reliability. In [22], an applicationmapping algorithm is proposed to minimize the effect of insertion loss. [23] introduced a methodology for the design space exploration of $\mathrm{ONoC}$ mapping solutions to minimize laser power consumption. Akram Reza proposed a chain of algorithms for multi-application mapping in ONoC $[24,25]$. So far, very few studies have been done on mapping that aiming to minimize the number of used wavelengths in WDM ONoC. However, the result of IP core mapping will affect the communication links in the network, which is related to the number of wavelengths utilized. It can be seen that designing a reasonable IP core mapping method may effectively reduce the network complexity while ensuring good network performance. Therefore, it is highly necessary to propose an IP core mapping approach that can minimize the number of wavelengths needed in WDM mesh-based ONoC.

DOI: $10.1587 /$ elex.17.20200045

Received February 9, 2020

Accepted February 17, 2020

Publicized March 10, 2020

Copyedited March 25, 2020 


\section{The proposed cluster WDM mesh-based ONoC architecture}

The mesh-based $\mathrm{ONoC}$ is architecture with regular topology [6]. It has many features that make it superior to other topologies. The simple and regular structure eases the design of the routing algorithm. Thanks to good scalability, it can be expanded into a larger network without changing other topological features. Therefore, mesh-based ONoC architecture has been widely used in the on-chip optical communication field.

In a typical mesh-based network, each router is connected to one IP core. This structure does not fully utilize the characteristics of WDM. To make full use of the wavelength resources available in the channel, a new architecture is proposed in this letter.

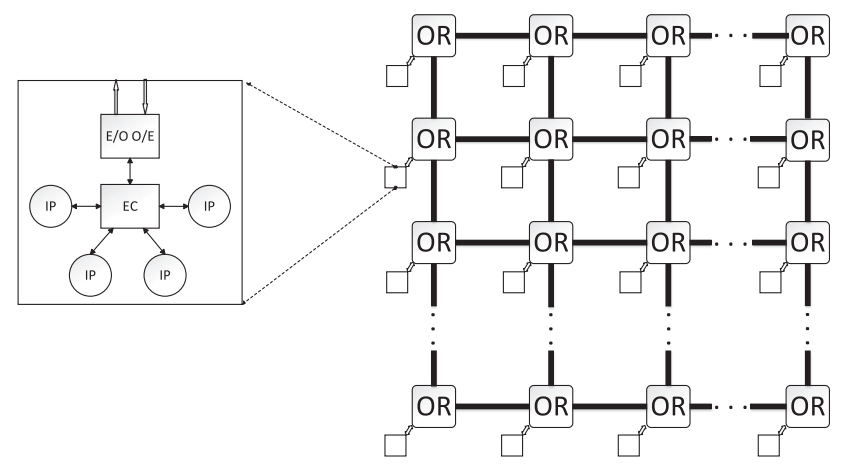

Fig. 1. Architecture of the proposed cluster WDM ONoC.

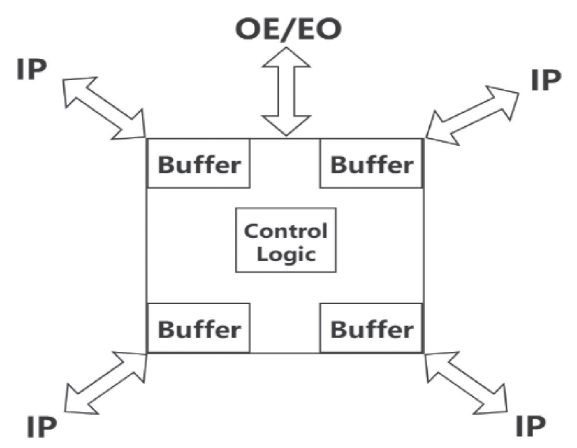

Fig. 2. Architecture of the electric crossbar

Fig. 1 depicts the proposed cluster WDM ONoC architecture. Each optical router (OR) is connected to a cluster IP cores group. The group consists of OE/EO converter, electrical crossbar (EC) and four IP cores. In this letter, a $5 \times 5$ electrical crossbar presented in [26] is adopted. The architecture of electrical crossbar is shown in Fig. 2. The electrical crossbar has a control logic and five ports, and the control logic is responsible for packet routing and arbitration. Five ports are connected to four IP cores in the group and an OE/EO converter. The ports contain the input and output channels, and the channels have buffers to store the packets temporarily. For the communication between IP cores within a cluster, packets are directly transmitted to the destination IP core through the electrical crossbar, and there is a three-cycle delay to transmit a packet, a single cycle for reading from a buffer, second cycle for arbitration and link traversal, last cycle for writing to a buffer of the destination core [27]. Therefore, packets within the cluster have a very short transmission time. For the communication between IP cores in different clusters, the packets are transmitted in the waveguides in the form of light.

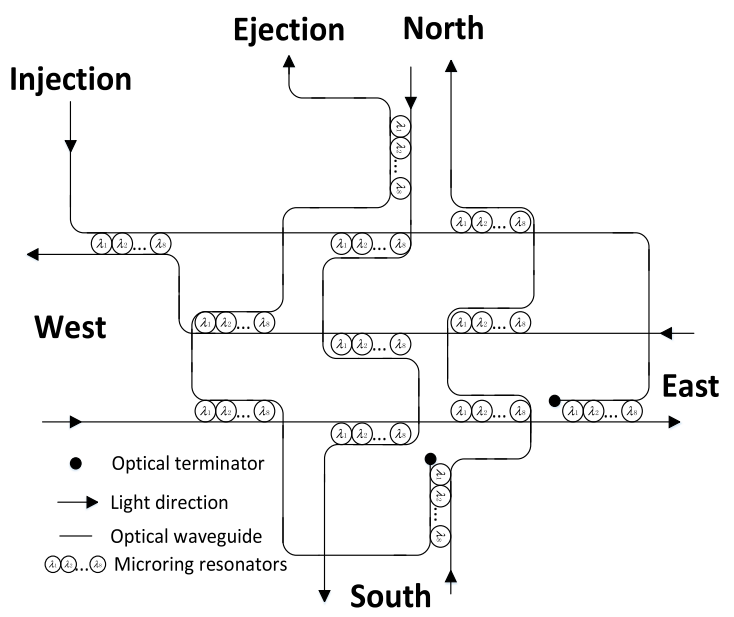

Fig. 3. Optical router of the proposed WDM ONoC.

This cluster architecture has three main advantages: (1) Optical communication in short distances does not have a prominent advantage compared to electrical communication [28]. Therefore, an electronic crossbar is used to route the data packets in short distance with a small delay and low energy consumption. In long distance, the data packets are modulated via the optical signals and are transmitted in the waveguide. In this way, the complexity of $\mathrm{ONoC}$ is reduced. (2) The architecture allows a router to be connected to multiple IP cores, where multiple communication links with different wavelengths may be accommodated in one waveguide. This can potentially improve the network throughput [16]. (3) In the current integrated optics processing technology, the volume of integrated optical devices on-chip is quite larger, and it is almost two orders of magnitude larger than that of electrical devices [29, 30, 31]. Cluster mesh-based $\mathrm{ONoC}$ uses fewer optical devices on-chip, thereby avoiding excessive area overhead. The optical router used in the architecture is responsible for routing optical data with multiple wavelengths. In this letter, a $5 \times 5$ optical router, presented in [1], is adopted as seen in Fig. 3.

\section{Mapping approach for WDM mesh ONoC}

In this section, an application-specific automated mapping approach to minimize the number of wavelengths for the cluster WDM mesh-based ONoC was described.

\subsection{Problem formulation}

For a mesh-based ONoC using a single wavelength, a photonic path need to be reserved to route the message. The optical routers along the photonic path are occupied. When one communication requirement needs to reserve an optical router occupied by another path, the blocking will occur at this optical router [32]. When the IP cores are 
mapped to the $\mathrm{ONoC}$, the communication links which include waveguide and intermediate optical router along the photonic path are determined. If an optical router belongs to more than one communication link, it will be blocked, and the number of blocks is equal to the number of communication links that the router belongs to. We use multi-wavelengths to ensure the router to be non-blocking, and the number of needed wavelengths should be the same as the number of blocks in the router. By figuring out the maximum number of blocks for all routers, the required number of wavelengths in network can be designated, and it equals to the maximum number of blocks.

To formulate the problem, the following definition is given: The application is modeled as a communication graph $G(C, A)$, in which each vertex $c_{i} \in C$ represents an IP core, and each edge $a_{i j} \in A$ represents the communication requirement between $c_{i}$ and $c_{j}$. The number of communication relations among cores represents the total number of communication requirements in communication graph. A topology graph is a directed graph $G(T, L)$, where $t_{i k} \in T(1 \leq k \leq 4)$ is a tile in the network, where $i$ is the optical router number, and $k$ is the tile number in the cluster. The $l_{i k, j k^{\prime}} \in L$ is a physical optical link between the tiles $t_{i k}$ and $t_{j k^{\prime}}$, and $t_{j k^{\prime}}$ is another tile which location is at the $k^{\prime}$ th tile in the cluster that connected to the $j t h$ optical router. Using these representations, the problem can be formulated as follows;

For a given $G(C, A)$ and $G(T, L)$, satisfying $\operatorname{size}(C) \ll \operatorname{size}(T)$, find a mapping function to minimize the number of used wavelength, and thus guarantee a nonblocking communication in the network. The fitness function can be expressed as

$$
\mathrm{F}=\max \left(\sum_{\forall c_{i} \neq c_{j} \in C} f_{o}\left(o_{m}(h), p_{i, j}\right)\right)
$$

subject to

$$
\begin{aligned}
& \forall c_{i} \in C, \quad \operatorname{map}\left(c_{i}\right) \in T, \\
& \forall c_{i} \neq c_{j} \in C, \quad \operatorname{map}\left(c_{i}\right) \neq \operatorname{map}\left(c_{j}\right),
\end{aligned}
$$

where $p_{i, j}$ is the routing path from core $c_{i}$ to $c_{j}$ in the network, and $f_{o}\left(o_{m}(h), p_{i, j}\right), h \in$ (east, west, south, north) indicates whether the out port $h$ of the $m^{\text {th }}$ optical router is used when $c_{i}$ communicates with core $c_{j}$, and it is calculated as

$$
f_{o}\left(o_{m}(h), p_{i, j}\right)=\left\{\begin{array}{l}
0, o_{m}(h) \notin \operatorname{link}\left(p_{i, j}\right) \\
1, o_{m}(h) \in \operatorname{link}\left(p_{i, j}\right)
\end{array},\right.
$$

where $\operatorname{link}\left(p_{i, j}\right)$ is a set of links used by cores $c_{i}$ and $c_{j}$.

\subsection{Mapping algorithm}

IP core mapping has two steps: in Step 1, the mapping results are attained by the mapping algorithm, and the fitness function is evaluated according to the estimation model. In Step 2, the solution is adjusted according to the algorithm rules. These two steps are repeated until the solution converges.

In this letter, a cluster WDM mesh-based ONoC mapping algorithm, based on particle swarm optimization (PSO), is proposed. In the PSO algorithm, the most optimal solution is found by a group of particles, where each solution is called a particle, and the quality of a particle is evaluated by its fitness. In the ONoC mapping problem, a particle corresponds to an ordering of cores onto the routers. After each iteration, the new solution is calculated by the following equation presented in [33]

$$
\begin{aligned}
& p_{k+1}^{i}=\left(s 1 * I \oplus s 2 *\left(p_{k}^{i} \rightarrow \text { pbest }^{i}\right)\right. \\
&\left.\oplus s 3 *\left(p_{k}^{i} \rightarrow \text { gbest }\right)\right) p_{k}^{i},
\end{aligned}
$$

where $p_{k}^{i}$ denotes the $i^{t h}$ particle of iteration $k . s 1, s 2$, and $s 3$ are the inertia, self-confidence, and swarm confidence values, respectively. The local best solution of particle $i$ is defined by pbest $^{i}$, and the global best solution of all particles is defined by gbest. According to (5), a new particle $p_{k+1}^{i}$ is created, and its fitness is evaluated. If it is better than the local best solution, the pbest ${ }^{i}$ will be updated. If it is better than the global best solution, the gbest will be updated. After a fixed number of iterations, the best solution is attained. The pseudo-code of the proposed algorithm is shown in Table I.

Table I. Mapping algorithm

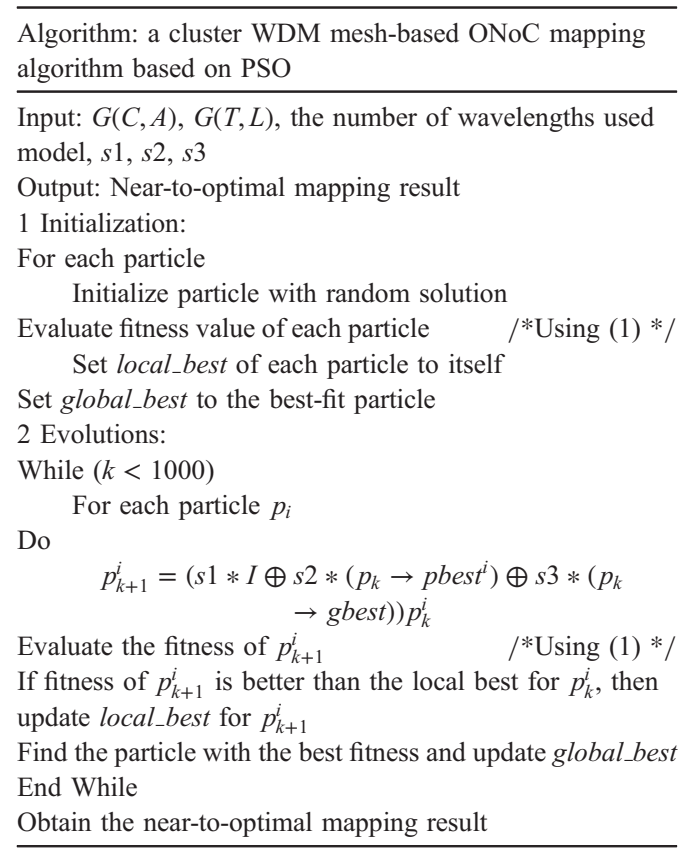

\section{Experimental results}

In this section, we present the experimental results of the mapping algorithm for the cluster WDM mesh-based ONoC.

\subsection{Number of wavelengths comparison}

In order to evaluate the effectiveness of the particle swarm optimization mapping (PSOM) algorithm proposed in this letter, we considered four typical video and image processing applications, namely MPEG4, WAVELET, VOPD, and DVOPD, whose communication graphs are shown in Fig. 4. The figure shows the number of cores and relations of communication among cores. As a contrast, the proposed PSOM is compared with random mapping (RM). The objective function value of the mapping algorithm is 
the number of wavelengths required to ensure a non-blocking communication. The size of the network is set to $3 \times 3$, which can accommodate 36 IP cores. The size of the network is defined as the number of optical routers in both directions. Fig. 5 shows the results of the mapping experiments for different applications.

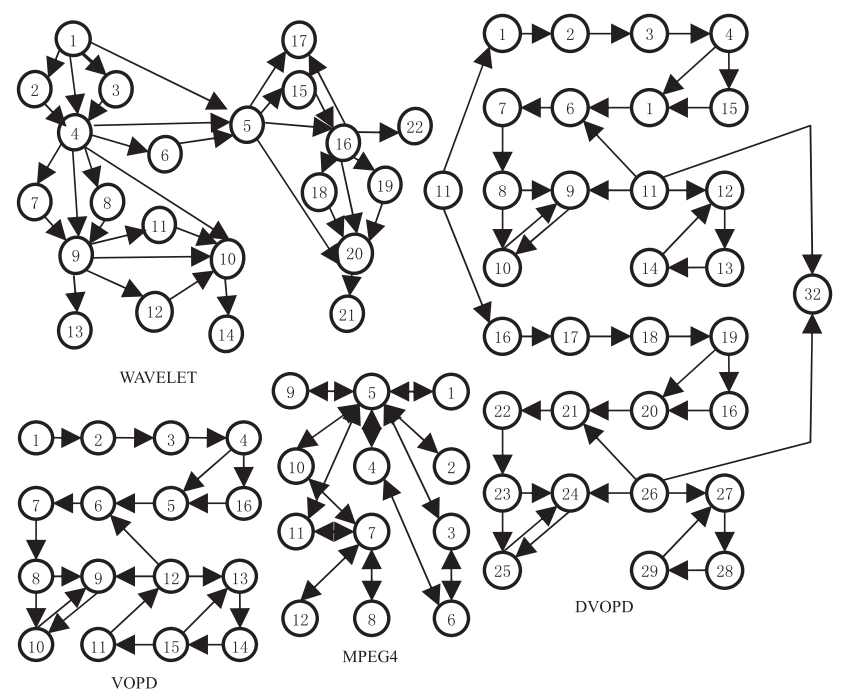

Fig. 4. Communication graphs for the four multimedia applications

We can see that the objective function value is significantly improved by PSOM. The number of wavelengths needed for MPEG4, WAVELET, VOPD, and DVOPD are reduced by $75 \%, 62.5 \%, 60 \%$, and $57.1 \%$, respectively. By observing the optimal solution, we found that the cores with more relations are more likely to connect to the same optical router. That is to say, more data packet flow is implemented by electric communication in one hop, and distant cores use waveguides to communicate. In this way, the $\mathrm{ONoC}$ can achieve non-blocking communication.

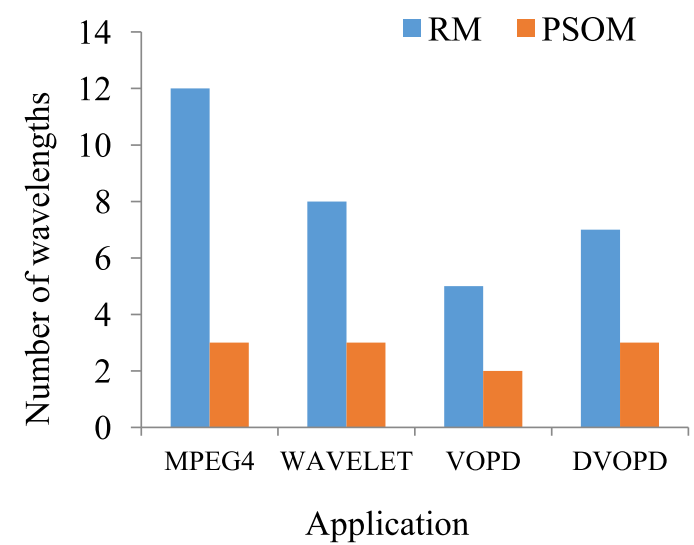

Fig. 5. Comparison of the number of wavelengths.

\subsection{Relationship between the network size and the number of wavelengths}

We have conducted an extensive analysis on the relationship between the network size and the number of wavelengths for an application with 16 cores. We considered four cases, which has 50,100, 150, 200 communication relations among cores, respectively. The size of the net- work is set to $2 \times 2,3 \times 3$ and $4 \times 4$. The number of wavelengths of the $2 \times 2$ network is compared to the $3 \times 3$ network and $4 \times 4$ network, as shown in Fig. 6 . As seen, while the number of communication relations among cores increases, more wavelengths are needed for each network. The reasons can be explained as follows. The total amount of network links resources including optical routers and waveguides is fixed for the network of same size, and congestion will deteriorate along with the increase of communication relations among cores. An alternative technique to avoid contention is increasing the number of wavelengths in networks, therefore more wavelengths are needed for the network with more communication relations.

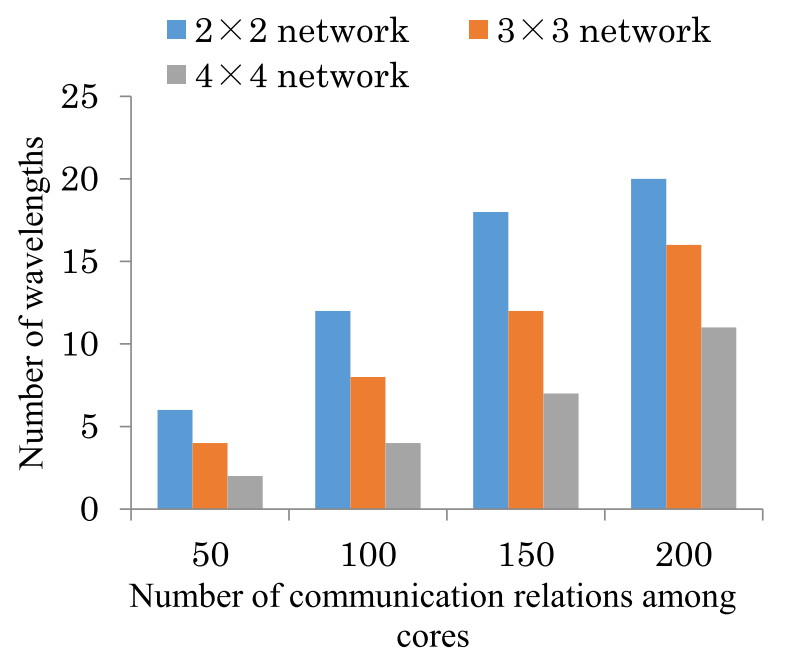

Fig. 6. Comparison of the number of wavelengths for varying network size.

As can be seen from Fig. 6, for the same number of communication relations, the larger the network size, the fewer wavelengths it needs. With the increase of network scale, there are more available optical routers and waveguides in $\mathrm{ONoC}$. Additional photonic paths can be reserved so that the packets can be distributed over the network. The probability that the optical router is occupied by multiple communication link is decreased. As a result, the number of blocks in the network decreases as the size of network increases. Therefore, fewer wavelengths are needed to relieve congestion. As revealed, the tradeoff between the network size and the number of wavelengths can be considered in the chip design flow. As the network becomes congested, it is feasible to reduce blocking by increasing network size or by increasing the number of wavelengths in the network.

\subsection{Communication simulation and comparison}

To investigate the network performance and the communication resources of the cluster WDM ONoC architecture (CWONoC) proposed in this letter, a network simulator is built in OPNET. Two types of ONoC are used for comparison. One is a cluster mesh-based $\mathrm{ONoC}(\mathrm{CONoC})$ proposed by [34], which adopts a single wavelength, and the other is a standard mesh-based WDM ONoC (SWONoC) [35], in which an IP core is connected to a router. The 


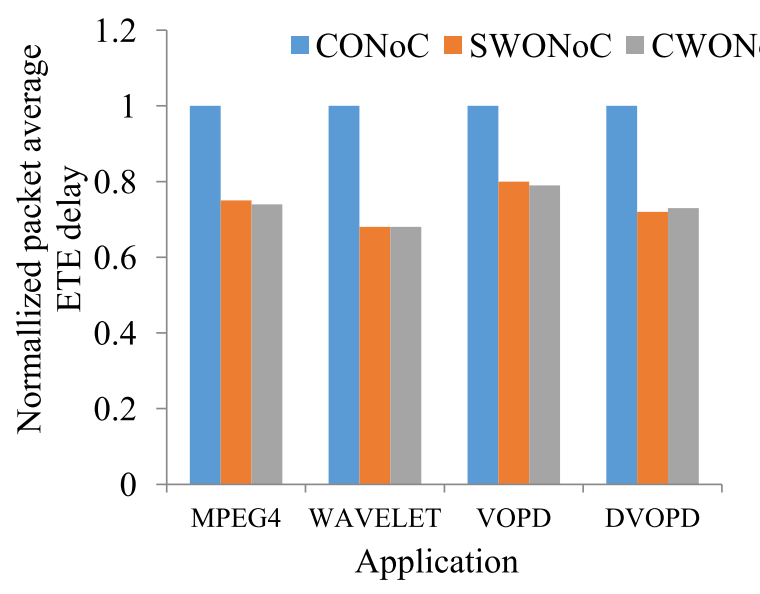

Fig. 7. Comparison of the normalized packet ETE delay.

packet size is assumed to be 2048 bits, and the optical data links bandwidth is set to $10 \mathrm{Gbps}$ for each wavelength [36].

We compared the normalized packet average end-toend (ETE) delay of CWONoC with CONoC and SWONoC for different applications. The results shown in Fig. 7 reveal that the ETE delay of SWONoC and CWONoC are almost the same, and were decreased by $25 \%, 35 \%$, $20 \%$, and $28 \%$ for MPEG4, WAVELET, VOPD, and DVOPD, respectively. For CWONoC and SWONoC, WDM is used to achieve a non-blocking network, where the ETE delay is almost the time of light transmission. For CONoC, since it performs circuit switching by a single wavelength, the delay is increased by blockage in the network.

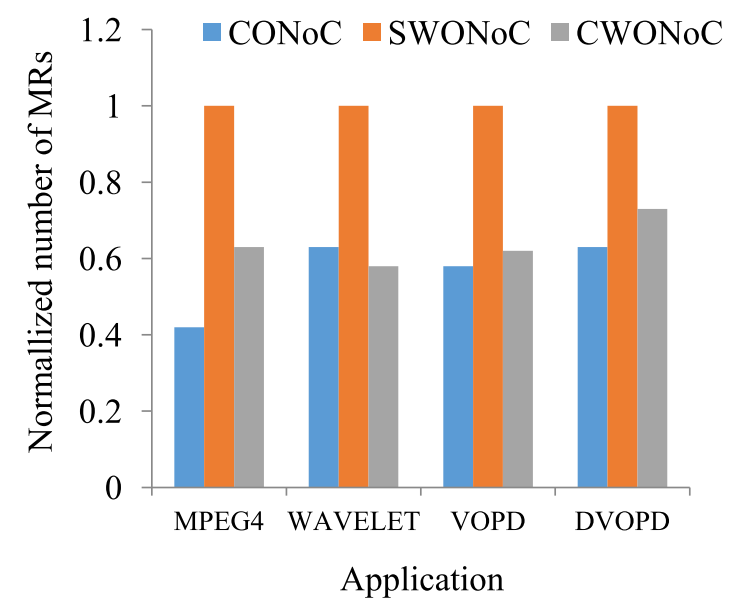

Fig. 8. Comparison of the normalized number of MRs.

The number of MRs needed is the most communication resource in $\mathrm{ONoC}$, and it has a big impact on the manufacturing costs of the chip [37]. The results provided in Fig. 8 show that SWONoC uses the most MRs. CWONoC and $\mathrm{CONoC}$ use fewer MRs since the cluster structure allows multiple IP cores to be connected to an optical router. The experimental results indicate that CWONoC improves the network performance without significantly increasing the communication resource overhead.

\section{Conclusion}

In this letter, cluster WDM mesh-based ONoC (CWONoC) is developed for a specific application with the aim of achieving non-blocking communications. To reduce the complexity in network and minimize the number of wavelengths needed, an IP core mapping algorithm, based on particle swarm optimization, is proposed. The experimental results show that our algorithm requires fewer wavelengths compared to random mapping. Furthermore, the tradeoff between the network size and the number of wavelengths is studied. We compared our structure with $\mathrm{CONoC}$ and SWONoC in terms of network performance, and the experiments revealed that $\mathrm{CWONoC}$ outperforms both, with an acceptable level of resource overhead.

For future work, we will do further research on the proposed ONoC. Because crosstalk has a severe negative effect on data transmission reliability of $\mathrm{ONoC}$, it should be included in the mapping algorithm as another metrics.

\section{Acknowledgments}

This work was supported by the Fundamental Research Funds for the Central Universities (No. NP2019102).

\section{References}

[1] R. Cao, et al.: "A crosstalk-aware wavelength assignment method for optical network-on-chip," IEICE Electron. Express 13 (2016) 20160821 (DOI: 10.1587/elex.13.20160821).

[2] E. Fusella and A. Cilardo: "Crosstalk-aware automated mapping for optical networks-on-chip," ACM Trans. Embed. Comput. Syst. 16 (2016) 1 (DOI: 10.1145/2930666).

[3] M. Petracca, et al.: "Photonic networks-on-chip: Opportunities and challenges," 2008 IEEE International Symposium on Circuits and Systems (2008) (DOI: 10.1109/ISCAS.2008.4542036).

[4] M. Yahya, et al:: "A low insertion loss design of rearrangeable hybrid photonic-plasmonic $6 \times 6$ non-blocking router for ONoCs," IEICE Electron. Express 16 (2019) 20190346 (DOI: 10.1587/elex. 16.20190346).

[5] K. Wang, et al.: "Layout optimization methodology for ring-based on-chip optical network," IEICE Electron. Express 16 (2019) 20190458 (DOI: 10.1587/elex.16.20190458).

[6] X. Yu, et al.: "A multi-wavelength communication strategy for 2D-mesh network-on-chip," IEICE Electron. Express 9 (2012) 706 (DOI: 10.1587/elex.9.706).

[7] X. Wang, et al:: "Universal method for designing non-blocking multicast-supported on chip optical router," IEICE Electron. Express 13 (2016) 20160667 (DOI: 10.1587/elex.13.20160667).

[8] A. Shacham, et al:: "Photonic networks-on-chip for future generations of chip multiprocessors," IEEE Trans. Comput. 57 (2008) 1246 (DOI: 10.1109/TC.2008.78).

[9] A. B. Ahmed, et al.: "Hybrid photonic NoC based on non-blocking photonic switch and light-weight electronic router," 2015 IEEE International Conference on Systems, Man, and Cybernetics (SMC) (2015) (DOI: 10.1109/SMC.2015.23).

[10] L. Bai, et al:: "A WDM multi-waveguide low blocking architecture for ONoC,” Opt. Laser Technol. 47 (2013) 355 (DOI: 10.1016/ j.optlastec.2012.08.024).

[11] S. Koohi and S. Hessabi: "All-optical wavelength-routed architecture for a power-efficient network on chip," IEEE Trans. Comput. 63 (2014) 777 (DOI: 10.1109/TC.2012.171).

[12] T. Zhou, et al.: "Rearrangeable-nonblocking five-port silicon optical switch for 2D-mesh network-on-chip," IEEE Photon. J. 10 (2018) 6601208 (DOI: 10.1109/JPHOT.2018.2841401).

[13] R. Min, et al.: "A universal method for constructing N-port nonblocking optical router for photonic networks-on-chip," J. 
Lightw. Technol. 30 (2012) 3736 (DOI: 10.1109/JLT.2012. 2227945).

[14] Y. Y. Xie, et al:: "Crosstalk noise analysis and optimization in $5 \times 5$ hitless silicon-based optical router for optical networks-onchip (ONoC)," J. Lightw. Technol. 30 (2012) 198 (DOI: 10.1109/ JLT.2011.2178396).

[15] S. V. R. Chittamuru and S. Pasricha: "Crosstalk mitigation for high-radix and low-diameter photonic NoC architectures," IEEE Des. Test 32 (2015) 29 (DOI: 10.1109/MDAT.2015.2414417).

[16] M. Nikdast, et al.: "Crosstalk noise in WDM-based optical networks-on-chip: A formal study and comparison," IEEE Trans. Very Large Scale Integr. (VLSI) Syst. 23 (2015) 2552 (DOI: 10. 1109/TVLSI.2014.2370892).

[17] I. Cerutti, et al.: "Scheduling in multi-wavelength ring-based optical networks-on-chip," J. Opt. Commun. Netw. 10 (2018) 322 (DOI: $10.1364 / \mathrm{JOCN} .10 .000322$ ).

[18] C. Wu, et al:: "An efficient application mapping approach for the co-optimization of reliability, energy, and performance in reconfigurable NoC architectures," IEEE Trans. Comput.-Aided Design Integr. Circuits Syst. 34 (2015) 1264 (DOI: 10.1109/ TCAD.2015.2422843).

[19] E. Fusella and A. Cilardo: "Minimizing power loss in optical networks-on-chip through application-specific mapping," Microprocess. Microsyst. 43 (2016) 4 (DOI: 10.1016/j.micpro.2016.01. 014).

[20] E. Fusella and A. Cilardo: "PhoNoCMap: An application mapping tool for photonic networks-on-chip," 2016 Design, Automation \& Test in Europe Conference \& Exhibition (DATE) (2016).

[21] L. Guo, et al.: "A novel IP-core mapping algorithm in reliable 3D optical network-on-chips," Opt. Switching Networking 27 (2018) 50 (DOI: 10.1016/j.osn.2017.08.001).

[22] S. Khoroush, et al.: "Application mapping in hybrid photonic networks-on-chip for reducing insertion loss," J. Supercomput. 74 (2018) 4647 (DOI: 10.1007/s11227-018-2458-7).

[23] E. Fusella and A. Cilardo: "Reducing power consumption of lasers in photonic NoCs through application-specific mapping," J. Emerg. Technol. Comput. Syst. 14 (2018) 23 (DOI: 10.1145/3173463).

[24] A. Reza: "Online multi-application mapping in photonic networkon-chip with mesh topology," Opt. Switching Networking 25 (2017) 100 (DOI: 10.1016/j.osn.2017.04.002).

[25] A. Reza and R. F. Mirzaee: "Non-preemptive offline multi-job mapping for a photonic network on a chip," Nano Commun. Netw. 11 (2017) 11 (DOI: 10.1016/j.nancom.2016.09.004).

[26] M. R. Seifi and M. Eshghi: "A clustered NOC in group communication," TENCON 2008 - 2008 IEEE Region 10 Conference (2008) 19 (DOI: 10.1109/TENCON.2008.4766825).

[27] R. Morris, et al.: "Extending the performance and energyefficiency of shared memory multicores with nanophotonic technology," IEEE Trans. Parallel Distrib. Syst. 25 (2014) 83 (DOI: 10.1109/TPDS.2013.26).

[28] Y. Ye, et al:: "3-D mesh-based optical network-on-chip for multiprocessor system-on-chip," IEEE Trans. Comput.-Aided Design Integr. Circuits Syst. 32 (2013) 584 (DOI: 10.1109/ TCAD. 2012.2228739).

[29] A. Biberman, et al.: "Broadband operation of nanophotonic router for silicon photonic networks-on-chip," IEEE Photon. Technol. Lett. 22 (2010) 926 (DOI: 10.1109/LPT.2010.2047850).

[30] D. V. Thourhout, et al.: "Nanophotonic devices for optical interconnect," IEEE J. Sel. Topics Quantum Electron. 16 (2010) 1363 (DOI: 10.1109/JSTQE.2010.2040711).

[31] M. Notomi: "Strong light confinement with periodicity," Proc. IEEE 99 (2011) 1768 (DOI: 10.1109/JPROC.2011.2123850).

[32] A. B. Ahmed, et al:: "Contention-free routing for hybrid photonic mesh-based network-on-chip systems," IEEE 9th International Symposium on Embedded Multicore/Many-Core Systems-onChip (MCSoC) (2015) (DOI: 10.1109/MCSoC.2015.24).

[33] P. K. Sahu, et al.: "Application mapping onto mesh-based networkon-chip using discrete particle swarm optimization," IEEE Trans. Very Large Scale Integr. (VLSI) Syst. 22 (2014) 300 (DOI: 10.1109/TVLSI.2013.2240708).

[34] W. Xu, et al.: "A blocking optimization method by convergence of cores for application-based optical circuit switched network-onchip,” Microprocess. Microsyst. 53 (2017) 168 (DOI: 10.1016/ j.micpro.2017.07.008).

[35] Y. Ye, et al:: "System-level modeling and analysis of thermal effects in optical networks-on-chip," IEEE Trans. Very Large Scale Integr. (VLSI) Syst. 21 (2013) 292 (DOI: 10.1109/TVLSI.2012. 2185524).

[36] X. Wu, et al.: "An inter/intra-chip optical network for manycore processors," IEEE Trans. Very Large Scale Integr. (VLSI) Syst. 23 (2015) 678 (DOI: 10.1109/TVLSI.2014.2319089).

[37] Y. Yang, et al:: "TAONoC: A regular passive optical network-onchip architecture based on comb switches," IEEE Trans. Very Large Scale Integr. (VLSI) Syst. 27 (2019) 954 (DOI: 10.1109/ TVLSI.2018.2885141). 